Gefässchirurgie 2013 $\cdot 18: 6-8$

DOI 10.1007/s00772-012-1090-8

Online publiziert: 13. Februar 2013

(c) Springer-Verlag Berlin Heidelberg 2013

\section{E.S. Debus}

Klinik und Poliklinik für Gefäßmedizin, Universitäres Herzzentrum, Universitätsklinikum Hamburg-Eppendorf
„Wenn man Chirurg ist, muss man jeden Morgen hungrig sein auf das, was man tun will."

(Chr. Barnard)

Dieser Ausspruch des südafrikanischen Pioniers der Herzchirurgie trifft nicht nur auf Chirurgen zu. Jeder, der etwas erreichen will, muss hungrig auf das sein, was vor ihm liegt. - In diesem Sinne möchte ich Sie als neuer Präsident der Deutschen Gesellschaft für Gefäßchirurgie und Gefäßmedizin - Gesellschaft für operative, endovaskuläre und präventive Medizin herzlich grüßen und zugleich einstimmen auf zwei Jahre, in denen viele Aufgaben auf uns warten.

Prof. Lang hat unsere Gesellschaft in den vergangenen zwei Jahren erfolgreich geleitet, dafür gebührt ihm unser Dank und Respekt. Die Projekte und Ziele, die in den vergangenen Jahren begonnen wurden, sollen erfolgreich weitergeführt werden. Kontinuität in der Arbeit ist eine der wesentlichen Voraussetzungen für Nachhaltigkeit. Diese Eigenschaft hat die Chirurgie von jeher ausgezeichnet, darf jedoch nicht Stillstand bedeuten. Die Entscheidung, auf Hergebrachtes zu verzichten und durch Neues zu ersetzen, muss sorgfältig getroffen werden. Unser therapeutisches Spektrum hat sich in den vergangenen 20 Jahren durch den enormen Innovationsschub in der Gefäßchirurgie dramatisch erweitert, aber trotz dieser Innovationen zählt das Operieren auch künftig zu den tragenden Säulen unseres Faches. In den vier Wänden des Operationssaales spielt sich dennoch nur ein Teil unserer Tätigkeit ab: Die Gefäßchirurgie ist heute vielseitig geworden, individueller in den Therapieentscheidungen und damit differenzierter auf den einzelnen Patienten ausgerichtet.

\section{》) Die Gefäßchirurgie ist heute differenzierter auf den einzelnen Patienten ausgerichtet}

Die zunehmende Implementierung endovaskulärer Eingriffe erfordert auch die Expertise unseres Assistenzpersonals. Mit der Etablierung der endovaskulären Assistentin (EVA) kommen wir dieser Forderung nach. Im Frühjahr wird diese berufsbegleitende Weiterbildung beginnen. Dies ist - neben der Gefäßassistentin DGG - die zweite, von der DGG initiierte Weiterbildung für ärztliches Assistenzpersonal. Mehr als in anderen chirurgischen Fächern ist der gefäßchirurgische Patient in seiner Gesamtheit als kranker Mensch anzusehen und zu behandeln. Nicht ohne Grund werden wir daher auch als Internisten unter den Chirurgen apostrophiert. Damit ist eines der zentralen Themen für die kommenden Jahre angesprochen: die Weiterbildung.

Hiermit meine ich zunächst nicht das Korsett, das uns das Arbeitszeitgesetz umschnürt und in dessen engem Rahmen es heute unmöglich geworden ist, einen $\mathrm{Pa}$ tienten von Anfang bis Ende zu betreuen wie es aber gerade in den klinischen Fächern notwendig ist. Wie viel Informationen gehen durch täglich mehrfache Übergabebesprechungen verloren und wie viel Informationsverlust endet für den Patien- 
ten schließlich fatal? - Wir wissen es nicht, aber es ist infrage zu stellen, ob diese engen zeitlichen Grenzen wirklich eine positive Innovation oder ein fataler Irrweg für Arzt und Patienten bedeuten.

Die Weiterbildungsordnung wird in den kommenden drei Jahren novelliert, fachliche Inhalte konkretisiert und damit das Berufsbild des Gefäßchirurgen von Morgen definiert. So viel scheint schon heute klar zu sein: Die Bundesärztekammer wird sich bei der Novellierung der Weiterbildungsordnung an europäischen Inhalten orientieren, nationale Alleingänge wird es deshalb womöglich nicht mehr geben. Umso mehr lohnt der Blick in die Weiterbildungsordnungen unserer europäischen Nachbarländer: Es ist erstaunlich, mit welcher Sorgfalt und Globalität in einigen Ländern bereits heute Inhalte definiert werden! Es wird da sinnvoll sein, schon frühzeitig den internationalen Schulterschluss zu suchen - die von uns bereits in der aktuellen Weiterbildungsordnung niedergelegten Fachinhalte und deren weitere Konkretisierung für die Zukunft ähneln den Strukturen vieler Nachbarländer bereits heute sehr.

Werner Lang hat in seiner Präsidentschaft die periphere arterielle Verschlusserkrankung in den Fokus gestellt. Dieses für uns zentrale Erkrankungsbild macht allein zahlenmäßig den Löwenanteil unserer Behandlungen aus. Umso wichtiger ist es, hier auch weiterhin den Fokus zu halten. $\mathrm{Zu}$ diesem Zweck werden wir von diesem Jahr an - ähnlich der AAA-Screeningkampagne - eine Awareness-Kampagne zur AVK auf den Weg bringen, die den Namen ACTION (Arteriosklerose und Circulation - Training und InfOrmations Netzwerk) trägt und neben der Peripherie auch die übrigen Gefäßprovenienzen beinhaltet, in denen die Gefäßverkalkung symptomatisch wird. Wir werden unsere Mitglieder mit unterschiedlichen Instrumenten, Pressemitteilungen, Patienteninformationen, Fachinformationen und Vortragsvorlagen für unterschiedliche Themenbereiche ausstatten, die über unsere Geschäftsstelle bezogen werden können. Unsere Gefäßtage der nächste Gefäßtag findet am 12.10.2013 statt - werden künftig unter dem Motto dieser Awareness-Kampagne stehen. Aber auch der von Prof. Eckstein initiierte AAAScreeningtag wird selbstverständlich bei- behalten. Das Datum für den nächsten AAA-Screeningtag ist der 27.04.2013. Bis heute sind wir noch auf dem Weg dahin, die Vorsorgeuntersuchung als gesetzliche Kassenleistung implementieren zu lassen hier ist ebenfalls Nachhaltigkeit gefordert, um dieses Ziel zu erreichen.

Die DGG ist eine wissenschaftliche Fachgesellschaft. Die Profilgebung in dieser Richtung wird ein weiterer wichtiger Schwerpunkt der kommenden Jahre sein. Wir haben bereits vieles erreicht, aber die Forschungs- und Lehrleistung an unseren Universitäten muss weiter intensiviert werden. Sie geht einher mit der Einrichtung von Kliniken und Abteilungen, womit auch eine Eigenständigkeit als Fachgebiet in Forschung und Lehre verbunden sein muss, analog dem Fächerkanon der chirurgischen Weiterbildungen. Ziel sollte es sein, an jeder Universität eine eigenständige Gefäßchirurgie vorzuhalten eine wichtige Grundlage, die dazu beiträgt, unseren wissenschaftlichen Nachwuchs auch in Zukunft heranzubilden.

\section{》Z Ziel sollte es sein, an jeder Universität eine eigenständige Gefäßchirurgie vorzuhalten}

Wir wollen dieser Entwicklung künftig auch dadurch vermehrt Gewicht geben, indem die DGG im Rahmen der Jahrestagung der Deutschen Gesellschaft für Chirurgie vom 29.4.-03.05.2013 in München eine Frühjahrstagung der DGG mit eigenem Gesicht unter dem Motto „GefäßChirurgie mit Leidenschaft und Augenmaß“ durchführt. Die gefäßchirurgischen Sitzungen werden künftig auf zwei Tage, in diesem Jahr dem 2. und 3.05.2013, konzentriert. Ziel ist es auch mit Blick auf die Einheit der Chirurgie, durch eine thematische Ausrichtung auf Weiterbildungsinhalte und State-of-the-Art-Vorträge vor allem unsere jungen angehenden Gefäßchirurgen zur Teilnahme zu motivieren. Hier findet sich die Plattform, auf der auch die Inhalte der basischirurgischen Weiterbildung vermittelt werden und alle anderen chirurgischen Fachgebiete vertreten sind. Ebenso werden wir unsere Kursangebote $\mathrm{zu}$ dieser Veranstaltung verlagern, um bei unserer spätsommerlichen Jahrestagung verstärkt Gewicht auf unsere wissenschaftlichen Themen zu legen.

Ein weiterer Baustein zur Etablierung der DGG als wissenschaftliche Gesellschaft stellt die Versorgungsforschung dar. Im internationalen Vergleich ist das deutsche Gesundheitswesen mit versorgungsrelevanten Daten nicht ausreichend abgebildet - umso wichtiger und vordringlicher erscheint uns diese Aufgabe. Die Bevölkerungsentwicklung und die damit zu erwartende massive Zunahme vaskulärer Erkrankungen erfordert dies ebenfalls. Mit dem Deutschen Institut für Gesundheitsforschung in der Gefäßmedizin (DIGG) haben wir die strukturellen Voraussetzungen und seit der konstituierenden Sitzung am 12.12.2012 in Hamburg auch die wichtigsten inhaltlichen und personellen Voraussetzungen dafür geschaffen, um eine umfassende epidemiologische Forschung zu vaskulären Erkrankungen und deren Therapie durchzuführen. Sie bauen wesentlich auf den Qualitätssicherungsmaßnahmen auf, die wir in Form von Therapieregistern umsetzen. Die ersten Register sind bereits erfolgreich initiiert worden (Aortenaneurysma), weitere werden in Kürze folgen (Varikosis und pAVK). Auch darüber hinaus sind Register in der Planung (z. B. Infektionsregister), die auch für Nicht-DGG-Mitgleider offen sind. Ziel ist es, eine möglichst vollständige Erfassung unserer Tätigkeiten mit ihren Ergebnisparametern zu erreichen. Dabei sind wir maßgeblich auf Ihrer aller Mithilfe angewiesen - je vollständiger die Datenerhebung ist, umso größer ist die Datenvalidität.

Bereits heute möchte ich Sie zu unserer diesjährigen Dreiländertagung - „Postmoderne Gefäßchirurgie“ - nach Linz einladen, die unter der Leitung von Dr. Franz Hinterreiter vom 03.-07.09.2013 stattfindet. Melden Sie Abstracts zu dieser Tagung an. Der Austausch mit unseren Kollegen und Freunden aus Österreich und der Schweiz ist immer ganz besonders inspirierend.

Mit der Übernahme der Präsidentschaft der DGG gebe ich nach 10 Jahren die Schriftleitung der Gefässchirurgie ab - eine Aufgabe, der ich mich mit viel Enthusiasmus und Herzblut immer gern gestellt habe. Sie endet nun turnusmäßig. Es ist mir eine besondere Freude, in Prof. 
Eckstein einen Nachfolger für diese Tätigkeit gefunden zu haben, dem das Prosperieren unserer Zeitschrift ganz besonders am Herzen liegt und der kontinuierlich, professionell und fachkundig neue inhaltliche und fachliche Schwerpunkte setzen wird. Ich weiß die Gefässchirurgie bei ihm in guten, sicheren Händen und wünsche ihm in unser aller Interesse für seine Aufgabe Erfolg und alles erdenklich Gute. Gleichzeitig danke ich an dieser Stelle allen, die sich als Autoren, Rubrikherausgeber, Herausgeber, Gutachter und Ratgeber an der Entwicklung der Gefässchirurgie beteiligt und mir als Schriftleiter mit Rat und Tat zur Seite gestanden haben, sehr herzlich. Ganz besonders danke ich meinem Freund und Stellvertreter in der Klinik, PD Dr. Axel Larena-Avellaneda, der in den letzten Jahren als Assistent der Schriftleitung wesentliches geleistet, mich vertrauensvoll und verlässlich unterstützt und mir viel Arbeit abgenommen hat. Der Erfolg unserer Gefässchirurgie ruht auf den Schultern von Ihnen allen!

Liebe Kolleginnen und Kollegen, ich freue mich auf die Präsidentschaft der kommenden zwei Jahre, auf die Gespräche mit Ihnen, den Austausch und vor allem die damit verbundenen Aufgaben. Ich danke Ihnen auf diesem Wege herzlich für Ihr Vertrauen, das Sie mir mit dieser verantwortungsvollen Aufgabe entgegenbringen. Gemeinsam werden wir auch in den nächsten zwei Jahren vieles erreichen. Packen wir's an!

Herzlich, Ihr

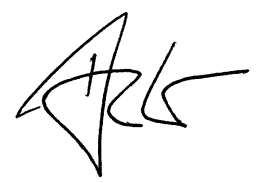

Prof. Dr. med. E.S. Debus Präsident der DGG

\section{Korrespondenzadresse}

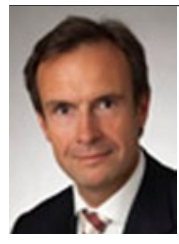

Prof. Dr. E.S. Debus

Klinik und Poliklinik für Gefäßmedizin, Universitäres

Herzzentrum,

Universitätsklinikum

Hamburg-Eppendorf

Martinistr. 52

20246 Hamburg

debus@uke.de

\section{Fritz Grupe \\ Traumjob Chefarzt}

Freising: STARK Verlagsgesellschaft mbH \& Co. KG 2012, 143 S., (ISBN 978-3-86668-611-3), Kartoniert, 19.00 EUR

Sobald man als Arzt bei einer der 17 Landesärztekammern gemeldet ist, also direkt nach der Approbation, erhält man regelmäßig das Deutsche Ärzteblatt. Sofort fällt der riesige Stellenmarkt ins Auge. Man gewinnt den Eindruck, dass man als Arzt kaum von Arbeitslosigkeit bedroht sein wird. Beruhigend ist das und man bemerkt darüber hinaus: Auch viele Chefarztpositionen werden dort ausgeschrieben. Insofern ist die Frage bei jungen Ärztinnen und Ärzten berechtigt: Kann auch ich Chefärztin oder Chefarzt werden? Wie sind die Chancen und was muss ich in den kommenden Jahren tun, um mich über meine medizinische Kompetenz hinaus, für eine Chefarztposition zu qualifizieren?

Der Autor Fritz Grupe berät private Klinikgesellschaften, öffentliche und konfessionelle Krankenhäuser bei der Auswahl von Chefärzten. In den vergangenen sechs Jahren hat er mehr als 200 Chefarztpositionen aller Fachrichtungen neu besetzt. Er beschreibt in seinem Buch sehr unterhaltsam und anschaulich, was ein Chefarzt von morgen können sollte und worauf sich ein potentieller Bewerber bei einem Auswahlverfahren einstellen muss.

Die Einleitung bietet einen kurzen Überblick über die Krankenhauslandschaft sowie die Herausforderungen für die Kliniken in den kommenden Jahren im Zuge der demographischen Entwicklung. Auch das geänderte Informationsverhalten seitens der Patienten hat Einfluss auf den Krankenhausalltag, da Patienten immer häufiger über Internet und andere Quellen einen höheren Gesprächsbedarf anmelden.

Die Gehaltsstrukuren von Ärzten werden übersichtlich dargestellt vom Assistenzarzt bis zum leitenden Oberarzt. Viele, dem erfahrenen Arzt geläufige Begriffe werden erläutert und kurz erklärt (DRG, CM-Punkte, CME etc.), sodass das Buch auch für Berufsanfänger verständlich geschrieben ist. Der Arbeitsablauf eines Chefarztes wird anhand eines fiktiven Beispiels realitätsnah dargestellt.
Vier Anforderungsprofile für eine Chefarztposition werden mittels realer Beispiele ausführlich vorgestellt, anhand derer man unschwer erkennen kann, dass alleine eine medizinische Expertise bei Weitem nicht ausreicht, um als Chefarzt akzeptiert zu werden. Ökonomische Verantwortung und strategisches Denken werden zunehmend wichtig. Fritz Grupe beschreibt anschaulich, wie eine Klinik bei der Suche nach einem geeigneten Bewerber vorgeht, womit ein Bewerber im Auswahlprozess rechnen muss und welche Gehälter gezahlt werden können. Wie kann ich Beruf und Privatleben als Chefarzt vereinen? Hier gibt es sowohl für einen potentiellen Bewerber, als auch für eine suchende Klinik wertvolle Hinweise.

Insgesamt gibt das Buch einen guten ersten Überblick für jungen Ärzte, die sich dem Berufsziel "Chefärztin/Chefarzt" realistisch nähern wollen. Es ist aber auch ein guter „last minute" Ratgeber, um sich professionell auf ein anstehendes Auswahlverfahren vorzubereiten.

Esther Wieland (Heidelberg) 